\title{
Novel application of simultaneous multi-image display during complex robotic abdominal procedures
}

Yanghee Woo ${ }^{1}$, Gi Hong Choi ${ }^{2,3}$, Byung Soh Min ${ }^{2,3}$ and Woo Jin Hyung ${ }^{2,3^{*}}$

\begin{abstract}
Background: The surgical robot offers the potential to integrate multiple views into the surgical console screen, and for the assistant's monitors to provide real-time views of both fields of operation. This function has the potential to increase patient safety and surgical efficiency during an operation. Herein, we present a novel application of the multi-image display system for simultaneous visualization of endoscopic views during various complex robotic gastrointestinal operations.

All operations were performed using the da Vinci Surgical System (Intuitive Surgical, Sunnyvale, CA, USA) with the assistance of Tilepro, multi-input display software, during employment of the intraoperative scopes. Three robotic operations, left hepatectomy with intraoperative common bile duct exploration, low anterior resection, and radical distal subtotal gastrectomy with intracorporeal gastrojejunostomy, were performed by three different surgeons at a tertiary academic medical center.

Results: The three complex robotic abdominal operations were successfully completed without difficulty or intraoperative complications. The use of the Tilepro to simultaneously visualize the images from the colonoscope, gastroscope, and choledochoscope made it possible to perform additional intraoperative endoscopic procedures without extra monitors or interference with the operations.

Conclusion: We present a novel use of the multi-input display program on the da Vinci Surgical System to facilitate the performance of intraoperative endoscopies during complex robotic operations. Our study offers another potentially beneficial application of the robotic surgery platform toward integration and simplification of combining additional procedures with complex minimally invasive operations.
\end{abstract}

Keywords: Multi-image display, Intraoperative endoscopy, Robotic surgery, Minimally invasive surgery

\section{Background}

The advantages of a robotic surgery platform have significant impact on the ability of surgeons to perform complex procedures. Currently, surgical robots provide advantages in two general areas: image-guided procedures and surgeon-controlled robotic operations [1]. Neurosurgery and orthopedics have successfully employed image-guided and computer assistance in their operations to increase both surgical accuracy and patient safety [2-4].

\footnotetext{
* Correspondence: wjhyung@yuhs.ac

2Department of Surgery, Yonsei University College of Medicine, 134 Shinchon-dong Seodaemun-gu, Seoul 120-752,

Republic of Korea

${ }^{3}$ Robot and MIS Center, Yonsei University College of Medicine, Seoul, Korea

Full list of author information is available at the end of the article
}

The use of surgical robots in general surgery has primarily been limited to surgeon-manipulated robotic operations, which have also demonstrated improved patient outcome [5-11]. As surgeons gain experience in robotic abdominal operations, surgeons are able to maximize the translation of technologies incorporated into the robot for patient benefit.

Complex robotic hepatobiliary, colon, and gastric surgeries have been successfully performed using the da Vinci Surgical System (Intuitive Surgical, Sunnyvale, CA) [12-16]. At times, additional intraoperative procedures to acquire more information on the patient's disease are required to supplement the preoperative work-up. For example, patients with gastrointestinal tumors may need intraoperative endoscopic localization of the tumors.

\section{Biomed Central}


During a liver resection for intrahepatic cholelithiasis common bile duct exploration, the use of a choledochoscope is required. These operations are being performed, in most cases, using additional monitors that make simultaneous examination of the intraabdominal working space and endoscopy difficult for a single surgeon. The surgical robot offers the potential to integrate multiple views into the surgical console screen, and for the assistant's monitors to provide real-time views of both fields of operation. This function has the potential to increase patient safety and surgical efficiency during an operation.

Our report presents a novel application of the da Vinci Surgical System's Tilepro multi-display system for simultaneous visualization of endoscopic views during various complex robotic gastrointestinal operations.

\section{Methods}

All operations were performed using the da Vinci Surgical System (Intuitive Surgical, Sunnyvale, CA, USA) with the assistance of Tilepro, multi-input display software, during employment of the intraoperative scopes. Tilepro software integrates into the robotic platform and permits the surgeon and operating room personnel to view up to three different images; that is, the operative view and images from two different video sources, simultaneously. Before each operation, the video output from the HDTVcompatible CV-180 Video Processor (Olympus, Tokyo, Japan), used for the three endoscopic procedures, was connected to the da Vinci Surgical System's console (Figure 1A). During intraoperative endoscopy, the surgeon activated the endoscopic images by turning on the multiinput Tilepro program at the surgeon's console using the video panel (Figure 1B). The surgeon was able to switch the endoscopic images on and off by tapping the camera foot pedal (Figure 1C).

\section{Operative procedures using Tilepro with intraoperative endoscopy}

Three robotic operations, left hepatectomy with intraoperative common bile duct exploration, low anterior resection, and radical distal subtotal gastrectomy with intracorporeal gastrojejunostomy, were performed by three different surgeons at a tertiary academic medical center. The patients' perioperative factors and clinicopathologic characteristics are shown in Table 1.

\section{Results}

\section{Colonoscopy during robotic low anterior resection}

A 66-year-old female patient with hypertension was diagnosed on routine screening colonoscopy with rectal cancer and subsequently underwent robotic low anterior resection with intraoperative colonoscopy. The preoperative colonoscopy revealed an ulcerofungating tumor located in the proximal rectum (Figure 2A). A
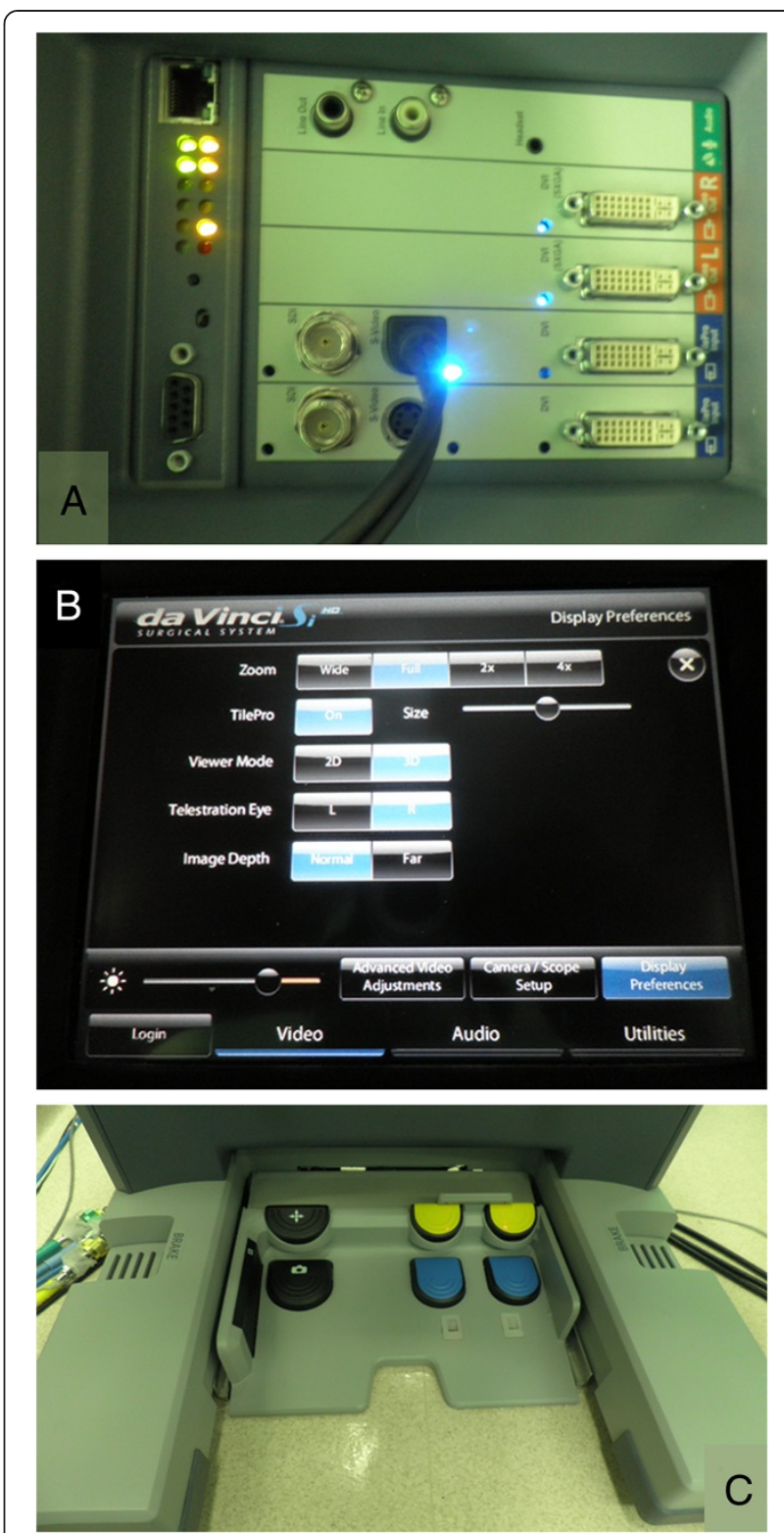

Figure 1 Tilpro $^{\mathrm{TM}}$ set up. (A) The posterior view of the da Vinci Surgical System input device for endoscopic video output. The surgeon can control the video input from the endoscopic procedures from the surgeon's console using the touch screen of the control bar (B) and the camera foot pedal (C).

computed tomography (CT) scan demonstrated no distant metastases.

Robotic low anterior resection was performed using the 6-port method as previously described [17]. In brief, after placement of the ports (one $12-\mathrm{mm}$ and five $8-\mathrm{mm}$ ports) and docking of the robot, a monopolar device, Cadiere forceps, and ultrasonic shears were used to mobilize the splenic flexure. The robotic arms were repositioned for the remainder of the operation. The surgeon mobilized the colon from the lateral peritoneal attachments by 
Table 1 Patient characteristics and perioperative factors

\begin{tabular}{|c|c|c|c|}
\hline Operation & Low anterior resection $\&$ colonoscopy & Radical gastrectomy \& gastroscopy & Left hepatectomy \& CBDE \\
\hline Age (years) & 66 & 41 & 57 \\
\hline Gender & Female & Male & Female \\
\hline Comorbidity & HTN & None & Hypothyroidism \\
\hline Operation time (min) & 396 & 200 & 465 \\
\hline Blood loss (cc) & 500 & 11 & 150 \\
\hline Complications & None & None & Fluid collect at hepatic resection line \\
\hline Length of stay (days) & 7 & 5 & 12 \\
\hline
\end{tabular}

division of the left paracolic gutter with careful preservation of the ureteral and gonadal vessels. Under direct 3-dimensional (3D) magnified vision, pelvic autonomic nerves and visceral fascia were preserved. The mesocolon was divided with ligation of the embedded feeding vessels, and the pericolic fat removed for preparation of resection of the sigmoid colon.

At this time, the assistant performed a colonoscopy (Olympus, Optical Co. Ltd, Tokyo, Japan) to localize the

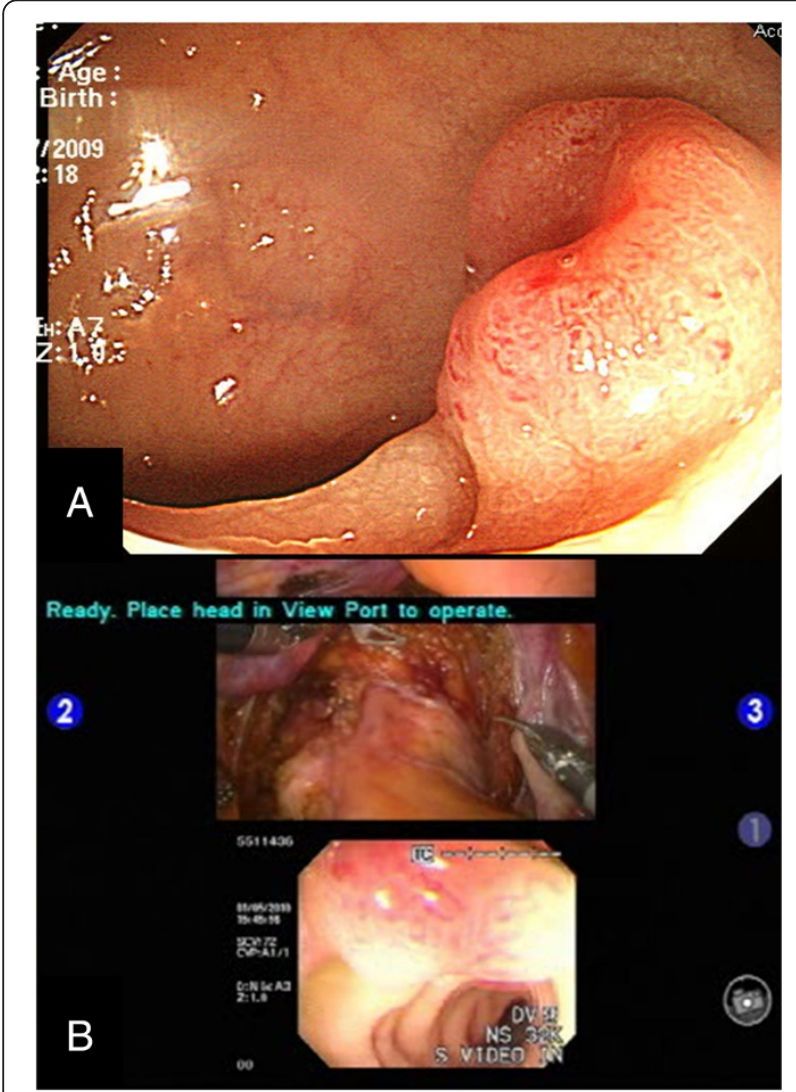

Figure 2 Preoperative colonoscopy and multi-input view of intraoperative colonoscopy. (A) The preoperative colonoscopic image. (B) Images from the intraoperative colonoscopy for tumor localization are visualized simultaneously with the intraabdominal extraluminal view for accurate determination of the distal rectal margin rectal lesion. The images from the colonoscopy were displayed on the surgeon's console along with the intraoperative extraluminal view of the rectum (Figure 2B). The tumor was found on the right side of the mid-rectum, and the distal margin was delineated. Aftern colonoscopic identification of the tumor was confirmed, simultaneous images on the screen of the previously cleared rectum permitted easy identification of the resection margin from the surgeon's console (Additional file 1). After confirmation of the rectal lesion colonoscope was removed since the patient did not required full colonoscopy.

The operation continued immediately after the colonoscope was removed. The distal margin was resected with an endolinear stapler, and the proximal colon exteriorized. A double stapling method using the CDH $33 \mathrm{~mm}$ (Ethicon Endo-surgery, Johnson \& Johnson, Cincinnati, OH, USA) was used for the colorectal anastomosis. The total operation time was 396 minutes. The patient was discharged 7 days after surgery without postoperative complications. Final pathological examination revealed a $2.2 \times 1.7-\mathrm{cm}$ moderately differentiated adenocarcinoma of the rectum that invaded the proper muscle and one of 24 lymph nodes was positive, staged as pT2N1M0, stage IIIA. The proximal and distal margins from the tumor were $12 \mathrm{~cm}$ and $3.3 \mathrm{~cm}$, respectively.

\section{Gastroscopy during robotic radical gastrectomy}

A 41-year-old male patient with no significant past medical history underwent robotic radical gastrectomy with gastrojejunal reconstruction for early gastric cancer. He was diagnosed with gastric cancer on screening esophagogastroduodenscopy, which was confirmed by biopsy as signet ring carcinoma. The lesion was identified at the angle of the lesser curvature (Figure $3 \mathrm{~A}$ ). A CT scan revealed neither enlarged intraabdominal lymph nodes nor distant metastatic disease.

Robotic radical gastrectomy was performed as previously described $[13,18]$. In brief, the patient was positioned in the supine position with the operating table placed in reverse Trendelenburg, and the trocars were inserted. After docking of the robotic arms and retraction of the liver, the proximal jejunum was identified 


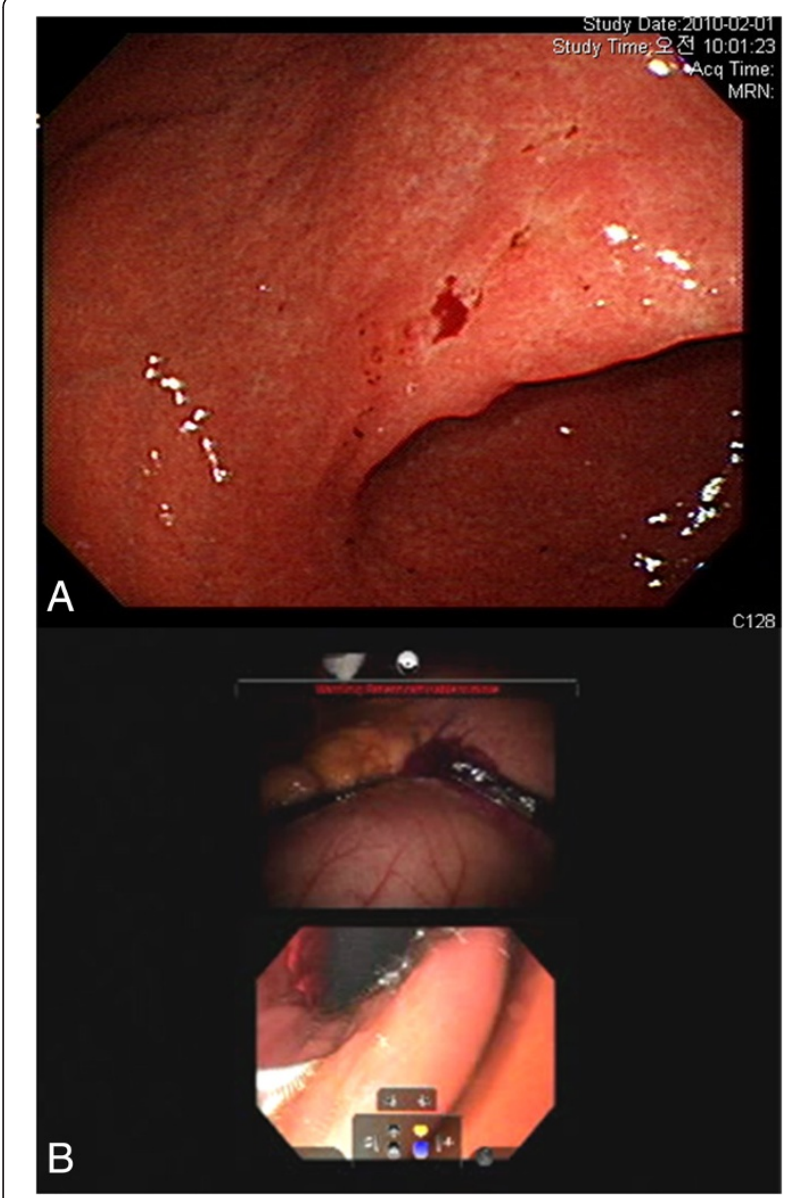

Figure 3 Preoperative upper endoscopy and multi-input view of intraoperative upper endoscopy. Gastric cancer was identified on preoperative upper endoscopy $(\mathbf{A})$. During the operation, the surgeon simultaneously viewed the endoscopic and intraabdominal images to accurately determine the proximal gastric resection margin (B).

and clamped to prevent small bowel distension during endoscopic air insufflation. Endoscopy was performed using a gastroscope (Olympus, Tokyo, Japan) to localize the tumor and determine the proximal resection margin. The endoscopic view was seen simultaneously with the intraoperative view of the stomach (Figure 3B). After endoscopic localization of the tumor, compression of the area of the tumor and the endoscopic light was used to define the proximal margin of resection. This view was facilitated by reducing the light from the robotic camera. The resection line was drawn on the external, anterior wall of the stomach (Additional file 2).

Robotic gastrectomy proceeded with partial omentectomy, ligation and division of the left gastroepiploic vessels, clearance of the greater curvature, and right-side dissection of the head of the pancreas with ligation and division of the right gastroepiploic vessels. After transecting the duodenum, dissection of the soft tissues surrounding the common hepatic artery and the celiac axis continued with identification, ligation, and division of the left gastric artery. The opening of the lesser sac and dissection along the retroperitoneal attachments to the stomach were completed with clearance of the lesser curvature. The previously determined proximal resection line was used as a guide to divide the stomach using an endolinear stapler. Since the intraluminal gastric lesion could not be palpated during robotic surgery, the use of an endoscope to identify the cancer assured an oncologically safe margin of proximal resection for proper intracorporeal anastomosis. Intracorporeal gastrojejunal reconstruction was performed with robotic assistance and stapler manipulation by the bedside assistant.

The total operation time was 200 minutes. The patient had an uneventful postoperative recovery and was discharged 5 days after surgery. Final pathological examination revealed a $3.2-\mathrm{cm} \times 2.5-\mathrm{cm}$ signet ring cell carcinoma invading the mucosa with no positive lymph nodes, staged as pT1aN0M0, stage IA. Proximal and distal margins from the lesion were $5.5 \mathrm{~cm}$ and $4.5 \mathrm{~cm}$, respectively.

\section{Robot-assisted common bile duct exploration during robotic left hepatectomy}

A 56-year-old woman with an 8-year history of hypothyroidism treated with methimazole underwent robotic left hepatectomy with intraoperative common bile duct exploration for multiple left intrahepatic duct stones associated with chronic cholangiohepatitis. A preoperative CT scan demonstrated multiple radioopaque stones in the left hepatic duct with ductal dilatation and parenchymal atrophy of the left lobe of the liver (Figure 4A).

Robotic left hepatectomy was performed as previously described [19]. In brief, the left lobe of the liver was freed from its attachments and adhesions due to chronic inflammation. The left hepatic artery was identified during hilar dissection and ligated. After identifying and preserving the venous branches to the caudate and Spiegel lobes, the left portal vein was ligated and divided. During parenchymal dissection, the left hepatic duct was identified and ligated. At this point, an incision was made proximal to the ligation of the left hepatic duct, and common bile duct exploration was performed.

The patient-side assistant introduced a 5-mm flexible choledochoscope (Olympus, Tokyo, Japan) into the abdominal cavity via the $12-\mathrm{mm}$ assist port. The surgeon sitting at the master console directed the choledochoscope using the first and third arms of the robot. The Cadiere forceps controlled by the third robotic arm held open the left hepatic duct, while the bipolar Maryland dissector was used to direct the choledochoscope into the open stump of the left hepatic duct and into the common bile duct. The view obtained from the choledochoscope was made available to the surgeon sitting at 


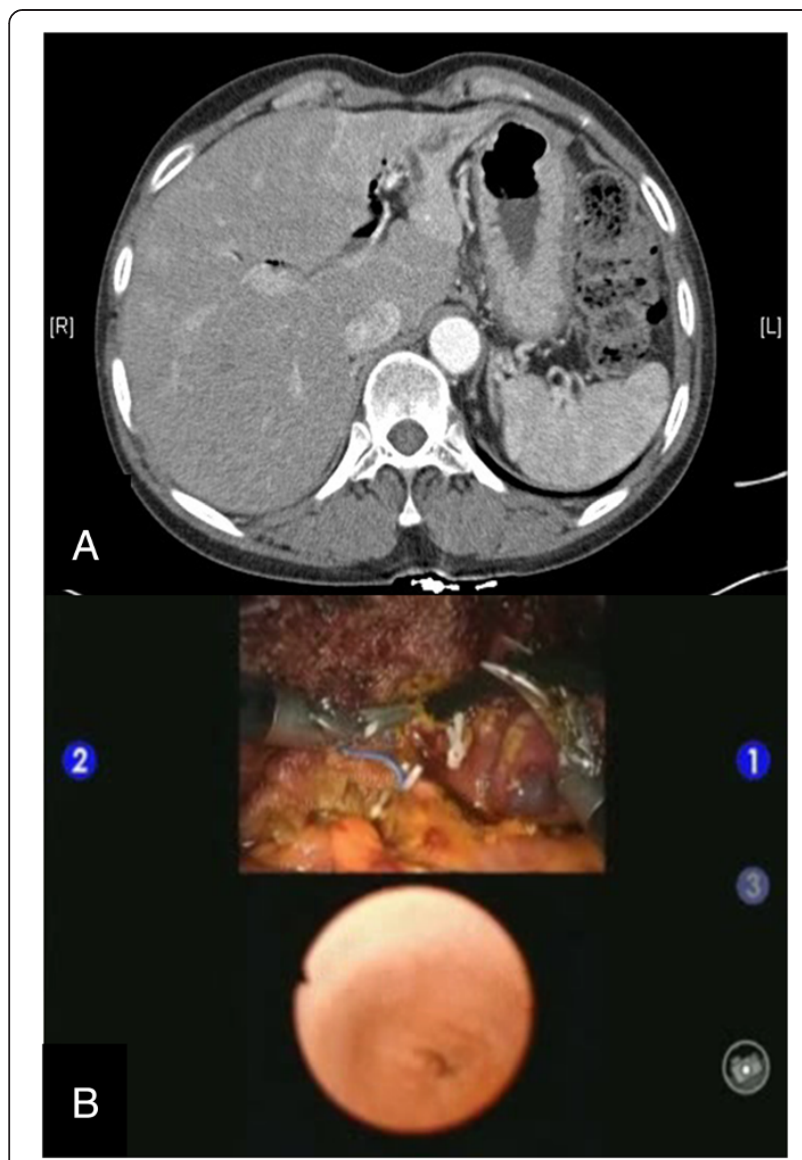

Figure 4 Images from a preoperative abdominal CT scan and multi-input view of intraoperative common bile duct exploration. A preoperative $C T$ scan of the patient with cholangiohepatitis shows intrahepatic ductal dilatation and left lobe atrophy (A). Intraoperative robot-assisted common bile duct exploration was facilitated by simultaneous visualization of the images from the choledochoscopy on the surgeon's console (B).

the console via the Tilepro multi-input system simultaneously with an intraoperative $3 \mathrm{D}$ view obtained by the da Vinci System's camera (Figure 4B). Robot-assisted intraoperative common bile duct exploration completely visualized the common bile duct and identification of the ampula of Vater but found no stones. Once the exploration was complete, the choledochoscope was removed, and the left hepatic duct was sutured closed using 5-0 prolene suture with robotic assistance (Additional file 3). Left hepatectomy was completed by identification and ligation of the left hepatic vein using a vascular endolinear stapler. The operation time was 465 minutes. The patient's postoperative course was complicated by fluid collection at the hepatic resection bed, which did not require invasive intervention. The patient was discharged 12 days after surgery. Final pathological examination revealed hepatolithiasis with pigment stones and chronic proliferative cholangitis. A follow-up CT scan obtained 1 month after surgery confirmed that no radioopaque stones were left in the common bile duct.

\section{Discussion}

The three complex robotic abdominal operations were successfully completed without difficulty or intraoperative complications. The use of the Tilepro to simultaneously visualize the images from the colonoscope, gastroscope, and choledochoscope made it possible to perform additional intraoperative endoscopic procedures without extra monitors or interference with the operations. The surgeons were able to continue with the operative procedures without distraction from their 3D view from the surgeon's console.

We describe a novel application of integrating intraoperative endoscopy during robotic abdominal operations using a multi-input display program available on the da Vinci Surgical System. Three different intraoperative endoscopic procedures were successfully delivered to the surgeon's console during robotic left hepatectomy, low anterior resection, and radical gastrectomy by employing the Tilepro, a multi-input display program. These three surgeries demonstrate the safety and feasibility of using the Tilepro to provide simultaneous, one-screen views of endoscopic procedures during robotic abdominal surgery, including rectal, gastric, and hepatobiliary operations.

Robotic operations have demonstrated safety and feasibility in various fields [20-22]. Robotic surgery offers the surgeon the potential to perform complex operations with increasing ease and precision as well as a quicker learning curve and adaptation to minimally invasive surgery over conventional laparoscopy [23,24]. Patients have benefited by decreased blood loss, decreased hospital stay, decreased pain, and increased satisfaction after their robotic procedures [5,8,9,25-27]. The advantages of robotic technology over conventional laparoscopic instruments have been predominantly attributed to the 3D operative view, tremor filter, 7 degrees of endowrist function, and control of four arms by the surgeon $[5,28]$.

As experience increases with the use of robotic surgical platforms, additional advantages are being realized. Recently, a group of urologists used Tilepro for imageguided surgery during robotic nephrectomies [29]. Patient specific information such as preoperative CT scans and intraoperative ultrasound was viewed intraoperatively on the surgeon's console to assist decision making during key portions of the operations. A multi-input display system permits the surgeon to view preoperative radiological images during surgery for guidance during the procedure.

Our study demonstrates another advantage of the surgical robotic platform: the ability of the surgeon to access various endoscopic images from the colonoscopy, gastroscopy, and choledochoscopy simultaneously during complex intraabdominal operations. This function shows the 
versatility of the multi-input display program and the ability of the surgeon to command a wider range of patient-specific information during the operation at his master console. No additional monitors are needed for the endoscopic views, not requiring the surgeon to stop the intraperitoneal portion of the operations. In fact, two views can be seen by the assistant and the scrub nurse on the assistant monitors, enabling everyone involved in the operation to share the same view. This function is especially useful during robot-assisted common bile duct exploration, where the surgeon was able to control the choledochoscope with the robotic arms without the need for another skilled assistant. The ability to perform both laparoscopy and choledochoscopy simultaneously using the current robotic surgical system demonstrates the potential for the development of a new integrated robotic surgery platform that allows the surgeon to simultaneously control two different procedures on one console. Since our study is an introduction of initial successful application of multi-image display system, we could not evaluate its clinical impact of by comparing with and without this new system. Apart from our study, this multi-image display system has innate limitations such as transmission failure due to a cabling problem [30].

\section{Conclusions}

We present a novel use of the multi-input display program on the da Vinci Surgical System to facilitate the performance of intraoperative endoscopies during complex robotic operations. Our study offers another potentially beneficial application of the robotic surgery platform toward integration and simplification of combining additional procedures with complex minimally invasive operations.

\section{Additional files}

Additional file 1: Video of Tilepro use for intraoperative sigmoidoscopy to identify the proximal margin of resection during robotic low anterior resection for colon cancer.

Additional file 2: Video of Tilepro use for intraoperative gastroscopy to identify the proximal margin of resection during distal gastrectomy for gastric cancer.

Additional file 3: Video of Tilepro use for intraoperative choledochoscopy to identify intrahepatic stones during left hepatectomy for chronic cholangiohepatitis.

\section{Competing interests}

The authors declare that they have no competing interests.

\section{Authors' contributions}

YW contributed to the acquisition of data, analysis and interpretation of the data, drafted the manuscript, revised it with critical input, and gave final approval of the version to the published. GHC contributed to the design of the study, the acquisition of the data, involved in drafting and critical review of the manuscript for intellectual content, and gave final approval of the version to be published. BSM contributed to the design of the study, the acquisition of the data, involved in drafting and critical review of the manuscript for intellectual content, and gave final approval of the version to be published. WJH contributed to the conception, design, writing, critical and intellectual input of the manuscript, acquisition and analysis of the data and gave final approval of the version to be published.

\section{Acknowledgements}

Written informed consent was obtained from the patient for publication of this manuscript and any accompanying images. This work was supported by a grant of the Korea Healthcare technology R\&D project, Ministry of Health, Welfare, \& Family Affairs, Republic of Korea (1320270). We acknowledge the assistance of BioScience Writers, LLC (Houston, TX, USA) in copyediting of the manuscript and corrections of English language usage.

\section{Funding source}

This work was supported by a grant of the Korea Healthcare technology R\&D project, Ministry of Health, Welfare, \& Family Affairs, Republic of Korea (1320270).

\section{Author details}

${ }^{1}$ Department of Surgery, College of Physicians and Surgeons, Columbia University, New York, NY, USA. ²Department of Surgery, Yonsei University College of Medicine, 134 Shinchon-dong Seodaemun-gu, Seoul 120-752, Republic of Korea. ${ }^{3}$ Robot and MIS Center, Yonsei University College of Medicine, Seoul, Korea.

Received: 2 August 2013 Accepted: 24 February 2014

Published: 15 March 2014

\section{References}

1. Singh I: Robotics in urological surgery: review of current status and maneuverability, and comparison of robot-assisted and traditional laparoscopy. Comput Aided Surg 2011, 16(1):38-45.

2. Rajasekaran S, Vidyadhara S, Ramesh P, Shetty AP: Randomized clinical study to compare the accuracy of navigated and non-navigated thoracic pedicle screws in deformity correction surgeries. Spine (Phila Pa 1976) 2007, 32(2):E56-E64.

3. Stulberg SD, Yaffe MA, Koo SS: Computer-assisted surgery versus manual total knee arthroplasty: a case-controlled study. J Bone Joint Surg Am 2006, 88(Suppl 4):47-54.

4. Lekovic GP, Potts EA, Karahalios DG, Hall G: A comparison of two techniques in image-guided thoracic pedicle screw placement: a retrospective study of 37 patients and 277 pedicle screws. J Neurosurg Spine 2007, 7(4):393-398.

5. Jacob BP, Gagner M: Robotics and general surgery. Surg Clin North Am 2003, 83(6):1405-1419.

6. Boggess JF, Gehrig PA, Cantrell L, Shafer A, Ridgway M, Skinner EN, Fowler WC: A case-control study of robot-assisted type III radical hysterectomy with pelvic lymph node dissection compared with open radical hysterectomy. Am J Obstet Gynecol 2008, 199(4):357. e351-357.

7. Atluri $P$, Woo YJ: Minimally invasive robotic mitral valve surgery. Expert Rev Med Devices 2011, 8(1):115-120.

8. Woo Y, Hyung WJ, Pak KH, Inaba K, Obama K, Choi SH, Noh SH: Robotic gastrectomy as an oncologically sound alternative to Laparoscopic resections for the treatment of early-stage gastric cancers. Arch Surg 2011, 146(9):1086-1092.

9. Song J, Kang WH, Oh SJ, Hyung WJ, Choi SH, Noh SH: Role of robotic gastrectomy using da Vinci system compared with laparoscopic gastrectomy: initial experience of 20 consecutive cases. Surg Endosc 2009, 23(6):1204-1211.

10. Horgan S, Galvani C, Gorodner V, Bareato U, Panaro F, Oberholzer J, Benedetti E: Robotic distal pancreatectomy and nephrectomy for living donor pancreas-kidney transplantation. Transplantation 2007, 84(7):934-936.

11. Giulianotti PC, Coratti A, Sbrana F, Addeo P, Bianco FM, Buchs NC, Annechiarico $M$, Benedetti E: Robotic liver surgery: results for 70 resections. Surgery 2011, 149(1):29-39.

12. de Souza AL, Prasad LM, Ricci J, Park JJ, Marecik SJ, Zimmern A, Blumetti J, Abcarian $\mathrm{H}$ : A comparison of open and robotic total mesorectal excision for rectal adenocarcinoma. Dis Colon Rectum 2011, 54(3):275-282.

13. Song J, Oh SJ, Kang WH, Hyung WJ, Choi SH, Noh SH: Robot-assisted gastrectomy with lymph node dissection for gastric cancer: lessons 
learned from an initial 100 consecutive procedures. Ann Surg 2009, 249(6):927-932.

14. Park JS, Choi GS, Lim KH, Jang YS, Jun SH: Robotic-assisted versus laparoscopic surgery for low rectal cancer: case-matched analysis of short-term outcomes. Ann Surg Oncol 2010, 17(12):3195-3202.

15. Kang CM, Chi HS, Kim JY, Choi GH, Kim KS, Choi JS, Lee WJ, Kim BR: A case of robot-assisted excision of choledochal cyst, hepaticojejunostomy, and extracorporeal Roux-en-y anastomosis using the da Vinci surgical system. Surg Laparosc Endosc Percutan Tech 2007, 17(6):538-541.

16. Chan OC, Tang CN, Lai EC, Yang GP, Li MK: Robotic hepatobiliary and pancreatic surgery: a cohort study. J Hepatobiliary Pancreat Sci 2011, 18(4):471-480.

17. Park YA, Kim JM, Kim SA, Min BS, Kim NK, Sohn SK, Lee KY: Totally robotic surgery for rectal cancer: from splenic flexure to pelvic floor in one setup. Surg Endosc 2010, 24(3):715-720.

18. Woo Y, Hyung WJ, Kim HI, Obama K, Son T, Noh SH: Minimizing hepatic trauma with a novel liver retraction method: a simple liver suspension using gauze suture. Surg Endosc 2011, 25(12):3939-3945.

19. Choi SB, Park JS, Kim JK, Hyung WJ, Kim KS, Yoon DS, Lee WJ, Kim BR: Early experiences of robotic-assisted laparoscopic liver resection. Yonsei Med J 2008, 49(4):632-638.

20. Ballantyne GH: Robotic surgery, telerobotic surgery, telepresence, and telementoring. Review of early clinical results. Surg Endosc 2002, 16(10):1389-1402.

21. Su LM: Robot-assisted radical prostatectomy: advances since 2005 . Curr Opin Urol 2010, 20(2):130-135.

22. Cho JE, Nezhat FR: Robotics and gynecologic oncology: review of the literature. J Minim Invasive Gynecol 2009, 16(6):669-681.

23. Pruthi RS, Smith A, Wallen EM: Evaluating the learning curve for robotassisted laparoscopic radical cystectomy. J Endourol 2008, 22(11):2469-2474.

24. Najarian S, Fallahnezhad M, Afshari E: Advances in medical robotic systems with specific applications in surgery-a review. J Med Eng Technol 2011, 35(1):19-33.

25. Maeso S, Reza M, Mayol JA, Blasco JA, Guerra M, Andradas E, Plana MN: Efficacy of the Da Vinci surgical system in abdominal surgery compared with that of laparoscopy: a systematic review and meta-analysis. Ann Surg 2010, 252(2):254-262.

26. Reza M, Maeso S, Blasco JA, Andradas E: Meta-analysis of observational studies on the safety and effectiveness of robotic gynaecological surgery. Br J Surg 2010, 97(12):1772-1783.

27. Waters JA, Canal DF, Wiebke EA, Dumas RP, Beane JD, Aguilar-Saavedra JR, Ball CG, House MG, Zyromski NJ, Nakeeb A, Pitt HA, Lillemoe KD, Schmidt CM: Robotic distal pancreatectomy: cost effective? Surgery 2010, 148(4):814-823.

28. Talamini MA, Chapman S, Horgan S, Melvin WS: A prospective analysis of 211 robotic-assisted surgical procedures. Surg Endosc 2003, 17(10):1521-1524.

29. Rogers CG, Laungani R, Bhandari A, Krane LS, Eun D, Patel MN, Boris R, Shrivastava A, Menon M: Maximizing console surgeon independence during robot-assisted renal surgery by using the Fourth Arm and TilePro. $J$ Endourol 2009, 23(1):115-121.

30. Bhayani SB, Snow DC: Novel dynamic information integration during da Vinci robotic partial nephrectomy and radical nephrectomy. J Robot Surg 2008, 2(2):67-69.

doi:10.1186/1471-2482-14-13

Cite this article as: Woo et al: Novel application of simultaneous multiimage display during complex robotic abdominal procedures. BMC Surgery 2014 14:13.

\section{Submit your next manuscript to BioMed Central and take full advantage of:}

- Convenient online submission

- Thorough peer review

- No space constraints or color figure charges

- Immediate publication on acceptance

- Inclusion in PubMed, CAS, Scopus and Google Scholar

- Research which is freely available for redistribution

Submit your manuscript at www.biomedcentral.com/submit
Biomed Central 